\title{
Tangence
}

\section{Un siècle de résistance à l'autobiographie}

\section{Philippe Lejeune}

Numéro 45, octobre 1994

Authenticité et littérature personnelle

URI : https://id.erudit.org/iderudit/025832ar

DOI : https://doi.org/10.7202/025832ar

Aller au sommaire du numéro

Éditeur(s)

Tangence

ISSN

0226-9554 (imprimé)

1710-0305 (numérique)

Découvrir la revue

Citer cet article

Lejeune, P. (1994). Un siècle de résistance à l'autobiographie. Tangence, (45),

132-146. https://doi.org/10.7202/025832ar d'utilisation que vous pouvez consulter en ligne.

https://apropos.erudit.org/fr/usagers/politique-dutilisation/ 


\section{Un siècle de résistance à l'autobiographie Philippe Lejeune}

L'autobiographie gêne. Elle gêne intellectuellement, esthétiquement, affectivement. C'est là ce qu'elle a de meilleur. La phrase du Monde épinglée par Daphni Baudouin, "les autobiographies des écrivains sont nécessairement des mythologies", parait droit sortir du Dictionnaire des idées reçues. Ce qu'on écrit dans Le monde aujourd'hui, on l'écrivait déjà dans la Revue des deux mondes des années 1880. Mais on oublie. Le mépris de l'autobiographie, aux mille facettes, toujours renaissant, fait trouver un charme toujours nouveau à ces platitudes anciennes.

L'autobiographie est méprisée par tous ceux qui savent ce qu'est vraiment l'art. Au seuil de ce petit essai, je voudrais faire entendre cette voix du mépris, sa violence. Dans un colloque universitaire (publié d'ailleurs par mes propres soins!) sur l'autofiction, on a pu lire récemment que l'autobiographie était "esthétiquement inepte", qu'elle traînait "un épais remugle de réalitée, qu'elle était la ajumelle difforme " de l'autofiction, le tout accompagné d'un avertissement diététique: "Qui se nourrit d'autobiographies risque de faire beaucoup plus de graisse que de muscle" 1 . Si on se tourne du côté des éditeurs, c'est le même recul effrayé (enquête de Lire, avril 1994): "L'abaissement de l'âge de la retraite nous coûte une forêt canadienne par an", "Tout y passe, le sida, le chômage, l'Algérie, parfois il s'agit de préoccupations plus intimes, comme ce récit des souffrances d'un hémorroïdaire reçu tout récemment". Tous les coups bas sont permis...

L'automne dernier, l'université de Yale organisait une commémoration de l'Histoire de la littérature française (1895) de Lanson. On nous demandait de faire le point: qu'est-ce qui a changé en cent ans? J'ai choisi de regarder ce qui touchait à l'autobiographie. Histoire d'un mépris (qui s'est métamorphosé), histoire d'une méconnaissance théorique (apparemment en voie de disparaître). Pendant le dernier quart de siècle (1969-1994),

1 Ces différentes formules sont extraites de la contribution de Maurice Mourier au recueil Autofictions $\mathrm{G}$ Cie, sous la direction de Serge Doubrovsky, Jacques Lecarme et Philippe Lejeune, RITM, nº 6, 1993 (p. 105, 104, 101, 103). 
mon histoire personnelle s'est développée dans le cadre de cette évolution. Je m'y suis donc replongé avec passion. J'ai relu, de Brunetière à Kundera, les pourfendeurs d'autobiographie. Commencé à réfléchir à l'incroyable retard de l'université française... J'ai pris des notes rapides, pour couvrir la toile. Elles sont comme le programme d'un livre à venir. Les voici, telles quelles. Je n'ai pas gommé mes humeurs. J'indique des pistes sans vraiment encore les suivre. Vue cavalière, avant-texte d'un livre qui peutêtre n'existera jamais... Contribution impromptue, sur le mode de la conversation, au débat ouvert avec bonheur par Tangence.

$$
* *
$$

En 1969, quand j'ai commencé à travailler sur l'autobiographie, j'ai été surpris du relatif vide de la littérature critique en France sur le sujet (Gusdorf excepté), alors que la littérature critique en allemand ou en anglais était déjà abondante. On dit pourtant que la France est le pays des Mémoires, et c'est en France qu'est apparu le texte fondateur que sont les Confessions de Rousseau. Mais justement, est-ce paradoxal? N'est-ce pas là l'explication? La tradition des mémoires aristocratiques et historiques a nui à la reconnaissance d'un genre apparemment voisin. D'autre part jusqu'à aujourd'hui inclus, les Confessions de Rousseau dérangent un grand nombre de personnes, et portent à l'incandescence les réactions hostiles au genre ${ }^{2}$.

En 1993, cela fait presque un quart de siècle que je travaille de manière descriptive sans doute, mais inévitablement normative, à l'étude de ce genre. Or si j'entends bien ce qui se dit autour de moi, ou bien je travaille sur un genre qui n'existe pas en dehors des définitions que j'en donne (car tout est fiction, qui naïvement s'ignore, ou qui s'avoue), ou bien au contraire il existe tellement, ce genre, qu'il est, disons-le carrément, la perte de la littérature moderne! un virus mortel qui tue l'art et la création! Alors je ne sais plus que penser... Sinon que peut-être la situation a peu changé depuis un siècle... Que l'histoire est très lente...

2 Sur la réception des Confessions au $\mathrm{xx}^{\mathrm{e}}$ siècle, voir Tanguy L'Aminot, Images de Jean-Jacques Rousseau de 1912 a 1978, Studies on Voltaire and the Eighteenth Century, no 300,1992 , p. 392-412 et 549-561. 
134

1887-1888: la France est en état de choc, ébranlée par une série d'attentats. Non, pas des attentats anarchistes, mais des attentats autobiographiques - c'est peut-être au fond la même chose. Depuis le début des années 1880 la France est enfin pour de bon en République, la période d'ordre moral qu'elle connaît depuis 1850 se termine. Et voilà qu'on publie - pas vraiment des autobiographies (La vie de Henry Brulard, publiée en 1890, a surpris certains, mais n'a choqué personne) -, mais des journaux qui, eux, font scandale. Une série de détonations qui ressemble à un complot: Amiel en 1883, Marie Bashkirtseff et Goncourt en 1887. La névrose, le flirt et l'orgueil, et des ragots de concierge, tout cela en librairie! Si vous y ajoutez la trilogie du communard Vallès, il y de quoi se demander où va la littérature, et où va la France.

Le débat a trouvé deux prestigieux hérauts: Anatole France, dans sa chronique du Temps (20 mars 1887), profite de la publication du journal des Goncourt pour lancer une sorte de manifeste en faveur de l'autobiographie: tout y est, sauf le mot. Son texte est si beau qu'on pourrait le graver sur le fronton de la bibliothèque d'Ambérieu-en-Bugey, siège, un siècle plus tard, d'une toute jeune "Association pour l'autobiographie et le patrimoine autobiographique", que j'ai contribué à fonder ${ }^{3}$. Voici son début provocant: "On reproche aux gens de parler d'eux-mêmes. C'est pourtant le sujet qu'ils traitent le mieux.... ${ }^{4}$. C'est presque un addendum à la Déclaration des droits de l'homme: le droit de dire sa vie... Toutes les autobiographies naissent libres et égales...

L'autre héraut, qui lui, bien sûr, sonne l'hallali du Moi, est Ferdinand Brunetière, dans sa chronique de la Revue des deux mondes (15 janvier 1888), intitulée "La littérature personnelle", un brûlot cicéronien d'une rare violence, un vrai rouleau compresseur de quarante pages ${ }^{5}$. Le point de départ est le même: la publication (pour lui scandaleuse et inepte) des journaux des Goncourt et de Marie Bashkirtseff. Comme Anatole France,

3 Cette association, fondée en 1992, rassemble des personnes qui aiment lire ou écrire des textes autobiographiques et s'intéressent à leur conservation. Elle publie un journal qui paraît trois fois l'an, La faute à Rousseau. Adresse: A.P.A., La Grenette, 01500 Ambérieu-en-Bugey, France.

4 Ce texte a été repris par Anatole France dans le volume I de La vie littéraire, en 1888.

5 Ce texte intitulé "La littérature personnelle" a été repris en 1897 dans ses Questions de critique. 
auquel, sans le nommer, il répond, il élargit immédiatement son propos, du journal à l'ensemble de l'écriture autobiographique. C'est un condensé de tous les arguments qu'on retrouve dans les polémiques d'aujourd'hui en France.

En lisant ces deux articles, un siècle après Rousseau, un siècle avant nous, j'ai eu l'impression de me trouver au centre d'un système. Ce n'est pas là un débat sur le genre, mais, au fond, c'est le genre lui-même, qui n'existe que dans l'espace créé par une telle tension... Le jour où l'acte et les textes autobiographiques seront unanimement acceptés, le genre sera mort. En 1990, j'ai publié un recueil de témoignages sur la pratique actuelle du journal intime, où j'avais permis à 47 personnes de présenter leur expérience. Ça s'appelait "Cher cabier...". C'était risqué: je savais que mon livre pourrait paraître... ennuyeux. Mais scandaleux, je n'y avais pas pensé. J'aurais dû. Car un nouveau Brunetière s'est levé, dans une petite revue d'avant-garde, pour nous incendier, moi et mes 47 diaristes, au nom de l'Art ${ }^{6}$. Baptême du feu, qui prouve que nous sommes bien vivants.

\section{La résistance à l'objet}

Mon premier travail consistera à analyser l'article de Brunetière, en voyant bien sûr ses failles, mais elles sont peu importantes par rapport à la solide argumentation d'ensemble qu'il construit. Elle n'est guère différente de celle qu'on trouve à la même époque chez Mallarmé ("la disparition élocutoire du poète" opposée à "l'universel reportage "), et qu'on retrouve aujourd'hui dans la bouche de toutes sortes de personnes très sensées, qui appartiennent aussi bien à l'avant-garde intellectuelle qu'au parti de l'ordre moral... Les clivages entre pro- ou anti-autobiographie ne recouvrent pas les grands clivages politiques. Souvent la ligne passe à l'intérieur des individus eux-mêmes, et pour un même individu, elle fluctue dans le temps - si j'en juge par mon propre cas...

Chez Brunetière je montrerai trois types de résistance:

- une résistance sociale (qui est peut-être la chose la plus datée dans son discours - quoique...?) : l'autobiographie est un

6 "Cher cabier... ", Gallimard, 1990. Marc Ligeray, "Lettre ouverte sur le journal intime", Recueil, Champ Vallon, $\mathrm{n}^{\circ}$ 19, 1991. J'ai répondu à Marc Ligeray dans le $n^{\circ} 21,1992$, de cette même revue. 
genre plébéien, un genre féminin, un genre enfantin..., de toute façon: un genre bas. Rousseau? "Le plus éloquent des laquais"! Brunetière parle du bout des lèvres de "cette petite peintresse de Marie Bashkirtseff". Il a l'allitération méprisante. Peur d'une invasion, d'une révolution... D'une démocratisation.

- une résistance éthico-psychologique: l'autobiographie est un vice, et une maladie. J'ai commencé à éplucher son texte pour trouver les sept péchés capitaux de l'autobiographie, j'en suis à cinq : paresse, orgueil, égoïsme, impureté, mensonge... Peur d'une corruption ou d'une contamination...

- une résistance esthétique: l'autobiographie est une facilité (l'art, lui, demande travail et élaboration), et surtout une absurdité (l'art suppose qu'on dépasse l'individuel pour arriver au général).

La violence de ce discours est liée à des peurs: peur de soi, et en même temps peur d'étouffement de l'Art, auquel on s'identifie, concurrencé en librairie par des livres qui, eux, ont du succès. Une des cibles, rarement nommée, puisqu'en fait c'est une partie de ces brebis égarées qu'on veut récupérer, c'est le public, qui se repaît de cette fausse littérature: on essaie de lui faire honte de ses goûts.

La démonstration de Brunetière a deux aspects: comme document, historique ou psychologique, l'autobiographie ne vaut rien (aveuglement des gens sur eux-mêmes, insincérité, orgueil, banalité, myopie); dans l'art, elle gâte tout, ou presque. En fait il n'y a que dans la poésie lyrique, dit-il, que le moi soit supportable, et que ce défaut tourne un peu à la qualité : les romantiques ont tous été de grands égoistes. Vigny est le meilleur, parce qu'il a su élever son moi jusqu'à une certaine généralité. Le roman personnel est très inférieur au vrai roman (Balzac). Et en critique et en histoire, le moi est totalement à exclure. Il a en particulier une page brillante qui réduit en poudre, avec un siècle d'avance, mon dernier livre, Le moi des demoiselles.

Il est amusant de voir comment l'univers mental de Brunetière est construit. D'un côté, il y a l'art, valorisé, avec cinq cases bien définies: théâtre, roman, poésie, critique, histoire - pas question d'ouvrir de nouvelles cases. De l'autre il y a, hors langage, la vie, qui est une pauvre chose, avec certes des "amours" et des "aventures" qui peuvent inspirer poètes et romanciers, mais surtout son long cortège d'échecs et de blessures d'amourpropre qui nourrissent de ressentiments et de vantardises la litté- 
rature du moi, et surtout une incurable banalité. Brunetière est un moraliste, il la connait par coeur, cette pauvre vie, pour lui elle n'a plus de secret. L'autobiographie est entièrement de ce côté-là: son seul rapport avec l'écriture, c'est de lui fournir un contenu informe, une motivation impure. Ce clivage absolu, postulé dès le premier paragraphe, dans un article dont la fonction normative est affichée, paralyse toute réflexion un peu ouverte, ou presque.

Un des points les plus curieux de ce long article est la position du problème historique. Brunetière écrit sous le coup d'un fait d'actualité. On a atteint le seuil de l'insupportable! La goutte d'eau qui fait déborder le vase!...C’est la décadence! (Air connu, cela se dit tous les dix ans depuis cent ans; dernière variation: Les testaments trabis de Milan Kundera, 1993...). Ce que Brunetière regrette, lui, c'est l'impersonnalité des grands classiques du $\mathrm{xVII}^{\mathrm{e}}$ siècle... - Mais sa raison d'historien le pousse à expliquer un phénomène que le moraliste en lui condamne. D'où une ou deux pages clairvoyantes, où il rapporte le développement de l'autobiographie au changement des structures sociales. Autrefois l'individu avait son identité garantie par une société hiérarchisée et stable. Maintenant, pour chacun, son identité est à conquérir. On est différent de son père, différent de son voisin. Mais de cette analyse il ne tire aucune conséquence. Il va à la ligne, retourne à ses colères, à ses anathèmes...

C'est curieux si on pense que ce même Brunetière, l'année suivante (1889-1890) dans son cours à l'École Normale, présentera un essai darwinien sur L'évolution des genres. Il n'a pas l'idée que, sur le long terme, il est peut-être en train d'assister (et de participer, au forceps!) à la naissance d'un genre... Quelques siècles plus tôt, le roman n'était-il pas né d'enrichissements et de métamorphoses à partir d'une pratique méprisée, bonne pour les femmes et les petits enfants?... Alors, qui nous dit qu'au $\mathrm{xxl}^{\mathrm{e}}$ siècle, l'autobiographie?...

\section{La construction de l'objet}

Bien sûr il faudra comparer l'article de Brunetière avec celui de France. On devine les oppositions. Mais on verra apparaitre un trait commun, qui sera le point de départ d'un autre aspect de mon étude. Sur une chose au moins Anatole et Ferdinand s'accordent : l'autobiographie... n'est pas de l'art! 
138

Le raisonnement de France est séduisant, et il se fonde apparemment sur une vérité d'expérience. On ne lit plus guère $L a$ nouvelle Héloïse ni les Natchez, on lira toujours les Confessions et les Mémoires d'outre-tombe. "Un poème, un roman, tout beau qu'il est, devient caduc quand vieillit la forme littéraire dans laquelle il fut conçu. Les ouvres d'art ne peuvent plaire longtemps... Or, des mémoires ne sont point des ouvres d'art. Une autobiographie ne doit rien à la mode. On n'y cherche que la vérité humaine...". Tout en louant le zèle d'Anatole France, il m'est difficile d'accepter l'idée que les Confessions et les Mémoires d'outre-tombe ne seraient pas des ouvres d'art!... J'ai écrit en 1975 Le pacte autobiographique pour montrer justement qu'il y a un art de l'autobiographie. Je me sentirais presque plus proche des propositions (qui pourtant me font horreur!) de Thibaudet lorsqu'il déclare, dans son livre sur Flaubert (1935): "L'autobiographie, qui paraît au premier abord le plus sincère des genres, en est peut-être le plus faux... C'est l'art de ceux qui ne sont pas artistes, le roman de ceux qui ne sont pas romanciers".

En fait le spontanéisme d'Anatole France comme le mépris de Thibaudet pour un erzatz aboutissent à exclure l'autobiographie du champ de l'art. Cette question des frontières de l'art m'a rappelé les grandes discussions théoriques qui ont eu lieu justement à la fin du $\mathrm{XIX}^{\mathrm{e}}$ siècle. Les pionniers d'une "science de la littérature" se heurtaient toujours au problème de la définition: où s'arrête la littérature? Je viens de relire une étude que j'avais faite en 1969 (et qui n'a pas été publiée) sur la définition de la littérature depuis Taine jusqu'en 1914. Je m'aperçois que le cas de l'autobiographie (auquel je ne pensais pas alors! et ces critiques non plus...) était virtuellement au cour de leur débat, qui opposait une vision restreinte de la littérature comme art à une vision élargie comme expression des mentalités et de la civilisation. Mon étude portait sur Taine (1863), Possnet (1886), Hennequin (1888, justement!), Lanson, Lacombe (1898), Renard (1900), Luchaire (1905)... Au fond, Anatole France se range tout de même du côté de la vision élargie... Mais la réponse à apporter, en ce qui touche l'autobiographie, est qu'il n'y a pas de frontière, qu'elle apparaît d'un bout à l'autre du spectre... Elle a ses propres règles de structuration, qui peuvent ou non se combiner avec ce qu'à cette époque, ou à la nôtre, on appelle "art".

En France, c'est seulement depuis les années 1970 que l'autobiographie a été intégrée au "canon" littéraire de l'école, à côté 
du roman, du théâtre, de la poésie... Elle a connu la même promotion que le roman policier, la science-fiction, le fantastique, la bande dessinée, tout ce qu'on appelle encore aujourd'hui "paralittérature". Des chapitres "autobiographies" apparaissent dans les manuels, on propose maintenant couramment pour le bachot des "groupements de textes" sur l'autobiographie, etc.

Pourquoi a-t-il fallu si longtemps? Que s'est-il passé pendant ce siècle qui nous sépare de Brunetière?

Il y a deux réponses possibles...

La première, évidente, est qu'en un siècle, l'autobiographie ellemême a changé. Les pratiques intermédiaires entre fiction et autobiographie se sont développées. Après le conformisme du XIX ${ }^{\mathrm{e}}$ siècle, la valeur d'acte de l'autobiographie est réapparue (Si le grain ne meurt, en 1926, L'âge d'bomme en 1939), tandis qu'émergait la revendication de l'autobiographie comme art (Biffures, 1948). Le journal intime, publié de son vivant par l'auteur même (Green, Gide), est entré dans les mours de l'édition: en particulier l'inauguration en 1939 de la Bibliothèque de la Pléiade par le Journal de Gide a contribué à légitimer le genre. À cette autobiographisation générale du champ littéraire a répondu, chez un certain nombre d'écrivains, une résistance proportionnelle. Résistance ambiguë, puisque souvent il s'agissait d'avoir l'autobiographie sans être eu par elle, si je puis dire. D'où le foisonnement de stratégies indirectes, d'où des tentatives de subversion, et du coup l'invention de formes nouvelles. Avec Michel Leiris et Georges Perec, l'autobiographie s'est déplacée de l'arrièregarde à l'avant-garde. Ajoutez à cela la vulgarisation progressive de la pensée psychanalytique, le développement de la parole autobiographique à la radio, après 1945, à la télévision, dans les années 1960, puis dans l'histoire orale (une date: 1966, commercialisation du magnétophone à cassettes). Il n'est pas étonnant que depuis cette fin des années 1960, dans la foulée d'une Révolution qui a surtout libéré la parole, on ait été sensible à ce bouillonnement. L'autobiographie est un peu soixante-huitarde... Il fallait donc boucher ce qui commençait à apparaître comme un trou béant dans le discours universitaire, en retard comme d'habitude sur les réalités ${ }^{7}$.

7 On verra un signe de cette nouvelle sensibilité dans l'ouvrage collectif dirigé par Jacques Bersani, La littérature en France depuis 1945, Paris, Bordas, 1970. Jacques Lecarme y met en évidence l'explosion autobiographique contemporaine. 
140

Mais c'est la seconde réponse qui me retiendra: examiner comment, au long de ce siècle, malgré tout, un discours critique sur l'autobiographie s'est peu à peu construit. Avec quel mal!... Les classements étaient déjà construits, il n'y avait pas de place pour elle... L'inertie universitaire...

L'autobiographie a d'abord été victime de la biographie. Les récits et journaux d'écrivains apparaissaient, avec leur correspondance, comme appartenant à la "vie", cette vie où l'on cherchait la clef ou le secret de l'œuvre. Ce statut de document les faisait passer du côté du non-texte. On s'y intéressait au coup par coup, dans le cadre de l'étude d'un écrivain particulier, pour l'information livrée. Jamais on ne les envisageait en série, comme des textes construits, obéissant à des règles communes, comme un genre. C'est le contraire de ce qui s'est passé en peinture. L'autoportrait peint a été très vite identifié comme un genre. Dès le $\mathrm{XVII}^{\mathrm{e}}$ siècle des amateurs ont commencé à en faire des collections, mettant en série des autoportraits de peintres différents. C'est que, bien sûr, la valeur informative était jugée secondaire: c'était d'abord de la peinture. En littérature, une ou deux œuvres seulement ont été intégrées au canon, mais comme des exceptions monstrueuses et inclassables: les Confessions et les Mémoires d'outre-tombe, présentés uniquement dans le cadre de chapitres consacrés à leur auteur, jamais comme les modèles ou les exemples-phares d'une pratique largement répandue. Les textes des autres écrivains s'évanouissent dans leur dossier documentaire, ce sont des "sources", au traitement parfois délicat. Le chapitre qu'André Maurois consacre à l'autobiographie dans Aspects de la biographie (1928) est à cet égard révélateur, analysant tous les types de "déformations" propres à cette source.

En fait, pendant le demi-siècle suivant (1890-1940), qui verra le développement foisonnant de grandes œuvres à dominante autobiographique (de Gide à Leiris, de Léautaud à Colette, de Céline à Malraux, etc.), la critique savante commencera timidement à construire une réflexion sur des objets partiels, et déjà anciens, avec un siècle de retard! Elle prendra conscience des innovations du siècle précédent, dans deux domaines :

- le roman personnel: il y aurait une étude épistémologique passionnante à faire sur la construction de cet objet, de Brunetière (1888) à Merlant (1905) et à Hytier (1928), tous penchés sur Senancour, Sainte-Beuve et Fromentin, au moment même où, 
sous leurs yeux, par ailleurs, créateurs et critiques construisent une nouvelle problématique (celle que j'ai appelée "espace autobiographique ")... Ce temps de retard de la formalisation théorique sur la pratique est inévitable. On en a un exemple frappant avec les débats actuels sur l'«autofiction": Serge Doubrovsky a inventé, en 1977, pour désigner une pratique qu'il croit nouvelle, un mot qui s'avère décrire fort bien... beaucoup de pratiques du demi-siècle précédent!

- le récit d'enfance: c'est seulement dans les années 1930 qu'on voit paraître une série de livres sur le récit d'enfance, essentiellement dans la fiction: Calvet (1930), Dupuy (1931), Toursch (1939)... Le titre du livre de Dupuy est révélateur: Un personnage nouveau du roman français, l'enfant. L'enfant n'attire l'attention critique (avec un demi-siècle de retard) qu'à partir du moment où il devient personnage principal de roman (c'est-à-dire à partir des années 1860-1870, Hector Malot, Daudet, Vallès et bien d'autres). Or il faut se souvenir que le récit d'enfance n'est pas né dans la fiction... mais dans l'autobiographie, et que c'est une invention de la seconde moitié du xviI ${ }^{\mathrm{e}}$ siècle (Rousseau, Rétif de la Bretonne, Mme Roland...). Il aura fallu un siècle et demi pour que ces textes extraordinaires entrent dans le champ de la réflexion critique, par raccroc, pistonnés par leurs arrière-petits neveux. C'est au moment où le récit d'enfant s'autonomise (en se détachant des autobiographies d'ensemble dont il était le premier acte) et se fictionnalise qu'il devient visible pour la critique...

Pendant ce demi-siècle (1890-1940), aucun livre d'étude ne sera publié en France ni sur l'autobiographie, ni sur le journal intime. Cela ne veut pas dire que rien ne s'écrive sur ce thème, loin de là : mais c'est du domaine du débat d'actualité, de la critique d'accueil, ou de la réflexion engagée des écrivains autobiographes eux-mêmes. Ces débats très vivaces n'arrivent guère à pénétrer dans une université relativement coupée de la culture contemporaine. Au début des années 1960 encore (je m'en souviens!) il était interdit de faire des recherches sur un écrivain vivant! On ne mettait jamais leurs cuvres au programme. Arrivant à Yale pour enseigner en 1963, j'ai été tout étonné de voir La jalousie (1957) au menu de "French 41"!

Les débats de ce demi-siècle toument autour du concept de sincérité, débat psycho-éthique, existentiel autant que littéraire. Il y a beaucoup de passion, des livres violents (Belphégor de Julien 
142

Benda, en 1917, La manie du journal intime et le roman autobiographique d'Émile Henriot en 1924), des essais (J. Rivière, De la sincérité envers soi-même, 1925), des bilans (J. Romains, La douceur de la vie, 1939), etc. C'est un universitaire français, mais émigré aux États-Unis, Henri Peyre qui a le premier dressé un tableau d'ensemble de cette problématique (Literature and Sincerity, 1963).

Je reviens en arrière. Jamais les autobiographies d'écrivains n'étaient constituées en genre digne d'étude; elles n'étaient pas non plus rapprochées des autobiographies de non-écrivains. Les Mémoires et témoignages n'avaient d'autre existence que d'être une des "sources" de l'histoire. Ces autobiographies ou journaux de gens ordinaires devaient fatalement passer inaperçus, tout simplement parce qu'ils n'étaient pas publiés, ou alors dans un contexte obscur... L'idée de l'autobiographie comme pratique culturelle large, idée qui commence (mais avec difficulté) à s'imposer aujourd'hui en France, était alors impossible.

Ce que les littéraires ne voyaient pas, d'autres auraient pu le percevoir, en particulier des historiens de la culture. C'est ce qui s'était passé en Allemagne avec le travail monumental de G. Misch, disciple de Dilthey. Dès le début des années 1900, il avait entrepris de réécrire l'histoire de l'humanité sous l'angle des progrès de l'autobiographie, donnant au genre les lettres de noblesse les plus anciennes. Mais rien de tel en France, où d'ailleurs Misch n'a jamais été traduit.

C'est seulement après la Seconde Guerre mondiale qu'une réflexion théorique sur l'autobiographie a vu le jour en France. Existentialisme et structuralisme, apparemment si contraires, l'ont successivement favorisée. L'existentialisme sartrien est une philosophie de l'individu et du projet, qui a une complicité particulière avec la démarche autobiographique. C'est dans cette atmosphère intellectuelle que se sont développées par exemple les recherches de Jean Pouillon, de Georges Blin sur Stendhal et de Jean Starobinski sur Rousseau. Mais les premières descriptions d'ensemble du genre sont venues des sciences humaines. Il faut saluer le travail de pionnier de Georges Gusdorf, avec La découverte de soi (1948) et Mémoire et personne (1950), puis son étude fondatrice de 1956, "Conditions et limites de l'autobiographie" 8 .

8 Publié dans Formen des Selbstdarstellung, Festgabe für Fritz Neubert, Berlin, Duncker und Humbolt, 1956. 
C'est donc un philosophe qui a le premier exploré l'histoire de l'autobiographie et sa problématique. Pour le journal intime, même situation: les deux premiers livres sur le genre sont le fait d'une psychologue adepte de la caractérologie, Michèle Leleu (1952), et d'un sociologue, Alain Girard (1963). Il faudra attendre 1976 pour que paraisse le premier livre écrit sur le journal par une spécialiste de littérature, Béatrice Didier, au moment où le genre aurait déjà pu fêter son bicentenaire... Et 1980 pour que soit fondé, par Claude Abastado, la première équipe de recherche littéraire sur les récits de vie, dans le cadre du Centre de Sémiotique Textuelle de l'Université Paris-X (Nanterre).

Signe des temps, ce Centre a été récemment débaptisé (il s'appelle maintenant RITM: «Recherches Interdisciplinaires sur les Textes Modernes»...). Son nom était devenu une gêne. Et pourtant il disait quelque chose d'historiquement important, il montrait le double visage du "structuralisme". Son opposition à l'autobiographie était apparemment catégorique: mort de l'auteur, mise en question du sujet. Les présupposés de l'autobiographie (référentialité du texte, possibilité de l'introspection, autonomie du sujet) n'étaient plus articles de foi mais occasions d'analyser des effets illusoires. On avait un peu pitié de ceux qui y croyaient encore. Les nouveaux savoirs regardaient le moi de haut. Roland Barthes, dans son Roland Barthes (1975), peint plaisamment cette mauvaise conscience qui habitait alors les modernes: plus je suis sincère, dit-il, plus je suis “interprétable" sous l'œil de l'Histoire, l'Idéologie, l'Inconscient... S'agissait-il d'une avancée des sciences humaines, ou d'un nouvel avatar d'une résistance centenaire? Au début des années 1980, cette résistance faiblira... Mais il y a bien eu avancée. À la différence de l'opposition morale et esthétique d'un Brunetière, celle du structuralisme a un côté constructif. Grâce à Benveniste, Genette et d'autres, il est devenu possible de parler de l'autobiographie comme d'un texte. D'en analyser les règles, d'en saisir les fonctions. Les nouveaux savoirs psychanalytiques et sociologiques, même s'ils mettent en lumière le côté illusoire des mythologies personnelles, permettent de comprendre la nécessité de ces illusions, et les voies qu'elles empruntent. Il n'est plus interdit de voir dans ce prodigieux travail que chacun fait pour construire son identité une forme d'art. L'université des années 1970 a pu rejoindre le Leiris des années 1940, celui de "De la littérature considérée comme une tauromachie" et de "Tambour-trompette ... 
144

Le groupe de Claude Abastado s'appelait "Récits de vie", et non "Autobiographie. Manière de rappeler que cet art n'était qu'une des manifestations possibles d'une pratique culturelle plus large. Et d'appeler à une collaboration interdisciplinaire. Elle est parfois difficile, parce que, malgré ce que j'ai dit plus haut de quelques pionniers, la plupart des spécialistes de sciences humaines en France aujourd'hui, psychanalystes, historiens, sociologues, restent animés d'une commune défiance à l'égard de l'écriture autobiographique. Ils préfèrent l'autobiographie orale, suscitée par eux, moins élaborée, plus ouverte à leur pénétration. Le sujet qui construit par écrit un discours sur sa propre vie est $a$ priori suspect... Trop coriace... Sous d'autres formes, la résistance Brunetière continue. Si bien que dans les années 1980, c'est de nouveau plutôt du côté de la philosophie qu'est venue une impulsion nouvelle, avec le travail de Paul Ricoeur, Temps et récit (1984-1986), et le concept d'identité narrative, qui permet de dépasser l'antinomie vérité/fiction. Après les années Gusdorf, couronnées par Lignes de vie (1990), voici peut-être les années Ricœur?...

J'arrêterai là cette petite histoire, qui arrive au présent. Au fond, nous espérons avoir dépassé à la fois le rejet moraliste de Brunetière et l'adhésion spontanéiste d'Anatole France, avoir commencé à comprendre... L'avons-nous fait? Rendez-vous, pour le savoir, à un prochain colloque, dans cent ans.

Je voudrais terminer ce bilan d'un siècle, sinon de résistance, du moins de réticence, de l'institution universitaire, par quelques considérations pédagogiques.

L'apprentissage du récit autobiographique (et d'ailleurs de toutes les formes d'écriture non critique) a toujours été réservé dans l'enseignement français à l'école primaire et au premier cycle du secondaire. Il est pratiquement abandonné dans le second cycle. La rédaction est un exercice considéré comme élémentaire, un peu puéril: "Vous raconterez votre premier chagrin". Dès qu'on est grand, on fait des dissertations. Donc Brunetière est très grand. Une des règles sacro-saintes de la dissertation est qu'on ne doit pas y dire "je", même quand on vous demande 
un avis personnel... Dans L'autobiographie en France, je disais "nous"; à partir du Pacte autobiograpbique, nous avons dit "je". Une fois, une revue m'a demandé de changer tous mes "je" en "nous". C'était une revue d'historiens. J'ai négocié... C'est vrai que ce n'était guère poli... Et c'est encore plus troublant si le "je" se charge d'un contenu réellement autobiographique, comme dans Le moi des demoiselles.

Quand on jette un regard en arrière sur ce siècle d'enseignement en France, un des paradoxes les plus amusants, c'est que cette institution rétive à l'autobiographie a abrité une immense population d'autobiographes. Ce sont les adolescent(e)s scolarisé(e)s (au lycée et à l'université) qui massivement tiennent des journaux... Et sans doute aussi un bon nombre de professeurs. Situation apparemment schizophrénique, qui fut la mienne, mais que j'ai vécue de manière très positive. Car du coup l'écriture de mon journal restait libre. Le retard de l'enseignement donnait à la littérature contemporaine, comme à l'écriture personnelle, une saveur de fruit défendu.

L'autobiographie commence maintenant à être intégrée: on étudie des cuvres, on fait des explications de textes, on discute la problématique, on ouvre des ateliers d'écriture... Le baromètre suprême, ce sont les programmes de l'agrégation. Seuls des textes à haute densité littéraire peuvent y figurer. Rousseau y a-t-il été pour les Confessions? Ce serait à vérifier. Chateaubriand, pour les Mémoires d'outre-tombe, oui. L'audace ne va pas plus loin. On n'y a, je crois, jamais vu Si le grain ne meurt, ni L'âge d'bomme. Récemment le jury a choisi les Souvenirs d'enfance et de jeunesse de Renan, un texte assez pompeux dans sa rhétorique, moralement conformiste, à contre-courant de la ligne inaugurée par Rousseau... Mais enfin c'est un effort... Peut-être un jour Les mots de Sartre?... L'agrégation est encore plus difficile à décrocher que le Nobel... En revanche, dans l'enseignement supérieur proprement dit, aujourd'hui des textes autobiographiques de toutes sortes sont couramment proposés à la lecture et à l'étude...

Il n'en est pas de même pour le journal intime. À ma connaissance, jamais aucun journal n'a figuré au programme de l'agrégation. Les journaux sont rarement étudiés comme textes en classe. De toute ma scolarité, je ne me souviens pas d'avoir jamais eu à expliquer un fragment de journal. Heureusement, pensais-je, car vraiment, qu'aurais-je bien pu en dire?... En tant 
146

qu'enseignant, je reconnais n'en avoir jamais proposé à mes étudiants. Je préférais, et préfère toujours, conserver cette barrière. $\mathrm{Ne}$ pas mélanger les genres. Voilà donc que la résistance a changé de camp. C'est la littérature en moi qui résiste à l'université. Le journal est mon genre réfractaire. Et s'il n'en reste qu'un, ce sera celui-là... 